\title{
Interactive Fabrication of CSG Models with Assisted Carving
}

\author{
Ammar Hattab \\ School of Engineering, Brown University \\ Providence, RI, USA \\ ammar_hattab@brown.edu
}

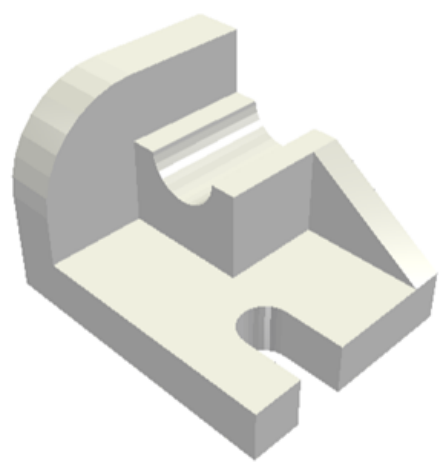

\author{
Gabriel Taubin \\ School of Engineering, Brown University \\ Providence, RI, USA \\ gabriel_taubin@brown.edu
}

Figure 1. We assist users in carving a physical CAD model. Given a target CAD model (Left). We generate a series of cutting instructions (Middle) and use a projector to project them to a block of material to guide the user in carving them. Until the user reaches the target physical replica (Right).

\begin{abstract}
We propose a method that helps an unskilled user to carve a physical replica of a 3D CAD model while only using manual cutting tools. The method starts by analyzing the input CAD model and generates a set of carving instructions. Then using a projector, we project the instructions sequentially one at a time to a block of material to guide the user in performing each of them. After each cutting step, we use the projector-camera setup to 3D scan the object after cutting. And automatically align the scanned point cloud to the CAD model, to prepare the position for the next instruction. We demonstrate a complete system to support this operation and show several examples manually carved while using the system.
\end{abstract}

\section{Author Keywords}

Interactive Fabrication; Assisted Carving; Constructive Solid Modeling (CSG); Spatial Augmented Reality.

\section{CCS Concepts}

-Human-centered computing $\rightarrow$ Human computer interaction (HCI);

Permission to make digital or hard copies of all or part of this work for personal or classroom use is granted without fee provided that copies are not made or distributed for profit or commercial advantage and that copies bear this notice and the full citation on the first page. Copyrights for components of this work owned by others than ACM must be honored. Abstracting with credit is permitted. To copy otherwise, or republish, to post on servers or to redistribute to lists, requires prior specific permission and/or a fee. Request permissions from permissions@acm.org.

TEI '19, March 17-20, 2019, Tempe, AZ, USA.

Copyright (C) 2019 Association of Computing Machinery.

ACM ISBN 978-1-4503-6196-5/19/03 ...\$15.00.

http://dx.doi.org/10.1145/3294109.3295644

\section{INTRODUCTION}

In the early stages of prototyping, users usually use digital and physical tools to create their design. They might start by creating a digital CAD model, then creating a physical mockup prototype by hand using foams. This allows them to feel the shape of the model and to make adjustments, before going back to digital again. The process of transforming a digital CAD model into a physical mock-up is very important at this stage. Some users use 3D printers for that, but they cannot use a prototyping material like foam or wood. CNC milling machines, on the other hand, could work on foam or wood, but they are usually large and expensive or they require some process planning to produce the 3D model from all directions. For this reason, a large amount of fabrication at this stage is done using hand tools.

Manually carving a model is an important skill for new designers. While many prefer digital design, there's something important lost when you cannot touch the thing you are making. Fabricating something with your hands is a very different cognitive process than drawing something on a screen. When designing models on the screen, it is necessary to make assumptions about the form and the function since the model is more abstract.

But the process of manually carving a digital model out of a block of material is still hard for novice users. Precise cutting is a painstaking job and figuring out the cutting steps in $3 \mathrm{D}$ is not trivial especially for complex models. Motivated by this problem, we present a method that can automatically analyze a CAD model and generates a series of manual cutting instructions. Then using a projector to project these instructions to 
guide the user in performing the cut until he reaches the target shape. The system has two main components; a method to generate manual cutting instructions, and a projector-camera calibrated setup to project those instructions, and to perform 3D scanning.

There are many 3D CAD representations, the most popular are boundary representations (B-Rep) and constructive solid geometry (CSG). In CSG, the CAD model is built using a tree of Boolean operations (like union, intersection, and subtraction) applied on simpler objects (like a sphere, cylinder, box, etc). In this work, we want to generate a series of subtraction instructions that remove parts of a block of material. So, we decided to use CSG for its volumetric representation. Starting from a block of material, each cutting instruction removes some part of the material until we reach the target model.

\section{RELATED WORK}

\section{Interactive Fabrication}

Fabrication machines were not fully automated when they were invented but involved a lot of human interaction. Programmers were required to write low-level commands to drive the machines. Conversational (CNC) allowed a higher level of interaction with the machines. While the (CAM) controlled machines became fully automated with time.

Many previous papers tried to increase the involvement of humans in the fabrication process, leading to what's called "interactive fabrication". The term "interactive fabrication" was first coined by [17] where they developed several interactive prototyping devices, "Constructable" [9] is another early break-through for making functional physical prototype interactively where users interact with the fabrication machine by drafting directly on the work-piece using a hand-held laser pointer, "Drill Sergeant" [14] project developed smart tools by augmenting common workshop tools (drill/driver, saw, router) with measurement and interactive feedback devices, and used them to provide the user with interactive in-situ step instructions, "Shaper Origin" [12] project combined manual and automatic tool positioning to precisely move a hand-held milling tool along a 2D path, and more recently in "RoMA" project [10] they combined augmented guidance with head display for interactive fabrication.

Within the interactive fabrication research a few previous papers proposed techniques to help novice users in carving, the closest to our work are the "Sculpting by Numbers" [11], "ShapeShift" [16], and Marner's work [7] where they used a projector to help users in sculpting clay or carving foam. The main difference between these papers and our work is that they provide a general guidance for the user by projecting the depth or the goal object on the remaining material and let the user figure out how to reach that goal. Whereas our method leads the user one step at a time through their process. Being more prescriptive than the other approaches, our method might be more suitable for novice users with little experience in carving or 3D. While the other papers might work better for more experienced users. In fact, this approach was one of the recommendations of the expert review in [7]. We also allow the user to move the model freely by hand to perform the cut, and then we automatically align the model for the next cutting step.

Besides using a projector, in the "FreeD" project [18] they proposed a hybrid carving technique, where they developed a hand-held digital milling device that helps the user in carving the 3D model. Although the carving could be faster than our work, they normally produce rougher output, which is more suitable for free-form sculptures rather than CAD models.

\section{Automatic Process Planning}

The method described in this paper starts by analyzing the CAD model and generates a list of cutting instructions, then it leads the user one step at a time to perform these cutting operations. While this might be suitable for beginner users, it's also very similar to the automated fabrication machines, where usually the CAD model is analyzed and a list of additive or subtractive instructions are generated and sent to the machine. In general, we call this process planning.

Additive machines like 3D printers, analyze the model by slicing it first and generate tool-paths from these slices. Milling machines, on the other hand, might require a more complex analysis, especially if the milling is to be performed from multiple directions, or in multiple stages using different machines. There has been a lot of work to automatically plan the fabrication process using different machines. Part of this work was focused on automatically discovering manufacturable features in the CAD model like holes, slots, and pockets, etc. See [5] for a survey of this work. The planning process might require segmenting the model into different surfaces, especially if a hybrid additive and subtractive fabrication is used as in [3].

\section{Spatially Augmented Reality}

In this paper, we use a projector to project cutting instructions onto a block of material. This creates an augmented reality on top of a real physical object. An approach that is called spatially augmented reality. See for example [2]. In general, this approach uses the projector intrinsic and extrinsic parameters for the rendering cameras, such that the rendering is performed from the same position and orientation of the projector with respect to the environment. The projector itself is considered as an inverse camera. Many previous papers used this approach, especially for educational purposes. Some papers used this approach for guiding the user to perform certain tasks, such as sculpting [16, 11], or painting [4], or placing objects in a certain position. The benefit here is that the user does not need to wear any special devices to see the virtual reality.

\section{METHOD}

\section{System Design}

In terms of hardware, the system is composed of a camera and a projector calibrated and fixed with regard to each other. The projector is used to project the cutting instructions on a block of material where it will illuminate the regions that should be removed by the user. The camera is used to capture the $3 \mathrm{D}$ shape of the object -together with the projector, see Figure 2.

In terms of software, the system is composed mainly of five components: 


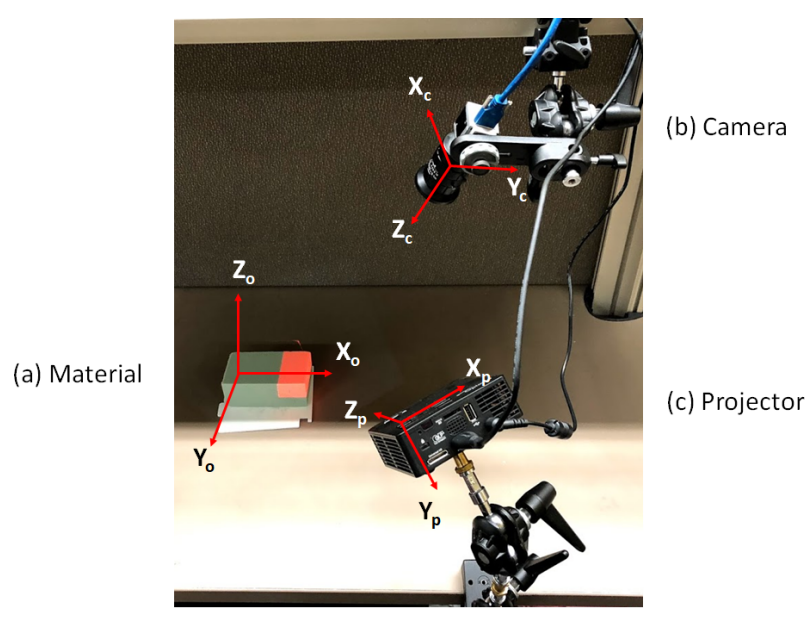

Figure 2. A projector-camera setup is used in this work

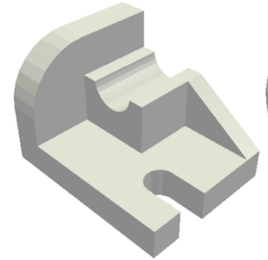

(a)

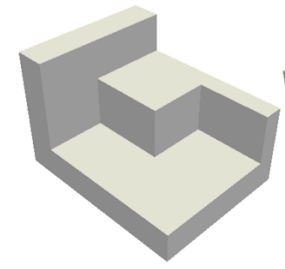

(d)

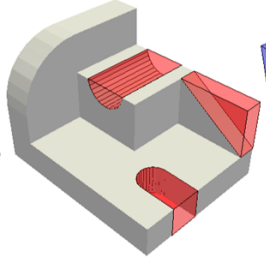

(b)

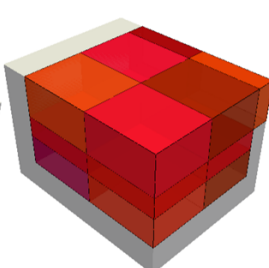

(e)

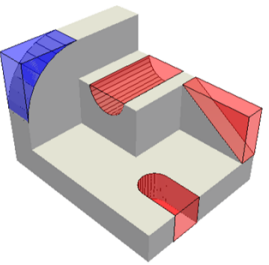

(c)

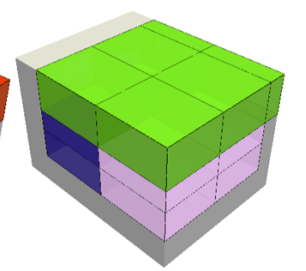

(f)
Figure 3. Method steps. (a) Given a CAD model. (b) We add negative objects instructions. (c) And we add simple positive objects instructions. This finishes stage 1 which results in a (d) union of bounding boxes object. (e) We construct a voxel grid of the remaining space. (f) And we segment the voxel grid by merging voxels.

\section{Instructions Generator}

The input to this step is a CSG CAD model, and the goal is to generate a series of cutting instructions to cut that CAD model out of a block of material. Each cutting instruction should remove a part of that block. And each cutting instruction should be simple enough to be done using hand tools. The union of all removed parts is equal to subtracting the CAD model from its the bounding box. So basically, to generate these cutting instructions, we turn the problem into a 3D segmentation problem of the negative space (The space resulting from subtracting the model from its bounding box). The algorithm performs the segmentation in two stages. See Figure 3 for a general overview of the algorithm.

In the first stage, we generate a cutting instruction for each simple geometry shape in the CSG tree (cube, cylinder, cone, etc). At first, for the negative shapes (shapes that are subtracted in the CSG tree), they are added directly to the list of instructions

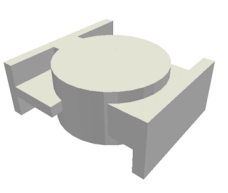

(a)

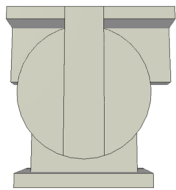

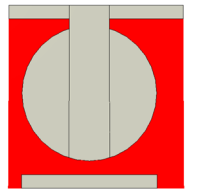

(b)

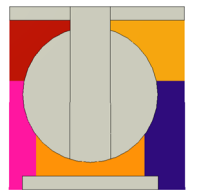

Figure 4. Segmenting the remaining volume by depth. (a) Bounding boxes object the result of stage 1. (b) Remaining volume shown in red color. (c) Segmenting the remaining volume by depth.

as the last cutting instructions. While for the positive shapes, if they are simple we add the negative space of the positive shape (its bounding box subtract the shape itself).

In the second stage, we start with the remaining volume to be cut, we define a voxel grid around it. Then we segment it by merging the small cells in the grid to get the remaining regions to be cut. We mark and exclude the filled voxels in this grid. Since the user usually starts cutting from the top, we need to look to the model from above. Starting from the top, for each remaining voxel, we compute the depth under this voxel (how many connected voxels under this voxel are marked as filled by the remaining volume). And, we run a region growing algorithm starting from that voxel (add the voxels that have the same depth to the same segment). After segmenting the voxels of certain z-level according to the depth, we start growing the segments down to the bottom levels. And we do so until no voxels are left. See Figure 4.

\section{D Scanning}

We need to capture the shape of the current object to find the correct alignment in order to project the next instruction. And to show the user how far he is from the target instruction. There are many 3D scanning techniques, but since we are using a projector to project the instructions, we can use it for 3D scanning as well. So, we used structured-light gray-code 3D scanning, and we used the method of [8] to calibrate the projector-camera setup.

Notice in Figure 2 that we have three coordinate systems, one for the camera, one for the projector and one for the 3D model. We choose to use the projector coordinate system as the main coordinate system and to convert all points and transformations to this coordinate system. Because the projector is the common thing between the two operations; 3D scanning with the camera, and 3D rendering with the 3D model. The point cloud resulting from scanning is converted to the projector coordinate system by a simple transformation using the rotation and translation matrices (we obtain them during the calibration process):

$$
P_{p}=R P_{c}+T
$$

While the $3 \mathrm{D}$ model is transformed into the projector coordinate system by the next $3 \mathrm{D}$ alignment step.

\section{D Alignment}

Fully carving a CAD model requires the ability to cut the model from several different orientations to cover the model details from all directions. Also, the user might want to hold the model in his hands or move it freely in any direction to use a specific tool. So, to allow a fluid process, we cannot 
restrict the model to a certain location or orientation. For this reason, after each cutting step, we must perform a 3D alignment step between the scanned 3D point-cloud and the current instruction 3D model. We used the iterative closest point (ICP) method to perform the automatic alignment [1].

The ICP method requires a good initial alignment to succeed. Otherwise, it could easily get stuck on some local minima. For this reason, after each cutting step, we ask the user to place the object roughly in the same position and orientation of the current instruction model. We do that by projecting a 2D guidance contour on the table and ask the user to place the object roughly on that contour. We generate that contour by slicing the bottom of the current instruction model. This usually provides a good initial alignment for the ICP to succeed. Otherwise, we allow the user to fix the initial alignment manually.

\section{Detecting Changes}

We also allow the user to use the result of 3D scanning to verify the previous cut. We calculate the distance between each point of the 3D scanned point cloud and the current instruction model, and visualize these distances using colors as in [6]. This visualization helps the user in fixing any deviation introduced during the last cut operation, unless if the deviation is an over-cut (which cannot be fixed easily in the context of carving). In the case of over-cut, the user might choose to update the current $\mathrm{CAD}$ model with the actual cut operation as detected by 3D scanning. To do that, we first find the changed regions in the 3D scanned point cloud by using the method of [6] where the user specifies a threshold distance after which the point is considered among the changed points. The minimum change that can be detected by the system depends on the resolution of the used 3D scanner. After detecting the changed regions, we use RANSAC method [13] to detect planes, cylinders or other basic shapes in the changed regions. Then, we use the detected shapes to update the shape of the actual CAD model using CSG operations. Sometimes, the system fails to detect the correct changed regions or the correct shapes due to noise or gaps in the 3D scans. In these cases, we allow the user to manually correct the changed regions or to ignore falsely detected regions.

\section{D Rendering and Projection}

Now, after alignment of the scanned point cloud and the current instruction model. The model is ready for projecting the next instruction. So, we use WebGL to render the next instruction while using the projector intrinsic parameters for the rendering camera [15], and we show the rendering on the projector screen. At the same time, we show a rendering of the whole model with the current instruction highlighted on the computer screen to allow the user to visualize it.

\section{EXPERIMENTS AND DISCUSSION}

\section{Carving Process}

The carving process starts from a block of material with the same dimensions as the bounding box of the CAD model, then we project a sequence of cutting instructions and ask the user to perform them, for each cutting instruction we need to perform the steps shown in Figure 5.

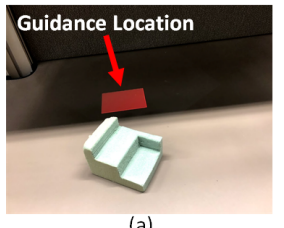

(a)

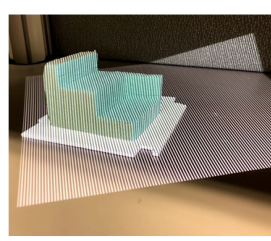

(d)

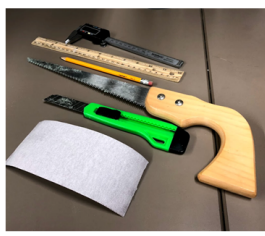

(g)

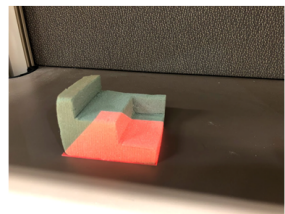

(b)

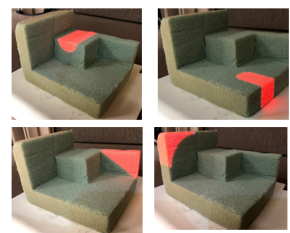

(e)

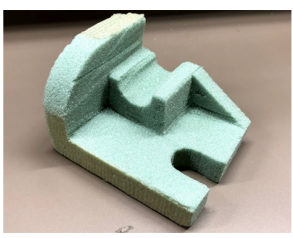

(h)

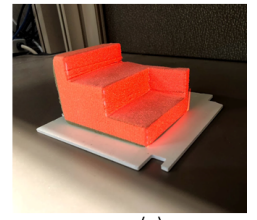

(c)

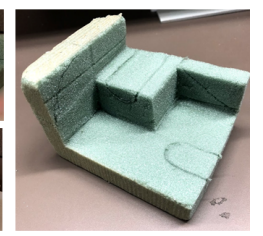

(f)
Figure 5. General process step. For a specific instruction, first, we find the best orientation. Then (a) We ask the user to place it in a guidance location. (b) The user roughly places the object in the guidance location (c) We project the whole object again for a better rough alignment. (d) We perform 3D Scanning. (e) We project the instruction(s). (f) The user marks the places to be carved. (g) The user performs the carving using hand tools. (h) result ready for the next instruction.

For each instruction, we need to find the best orientation to show it to the user that gives the best projector visibility. We simply want to choose from the six basic orientations; front, back, left, right, top and bottom. We do so by shooting rays starting from each polygon on the surface of the instruction in each of these directions. And choosing the direction that gives the best visibility (gives a larger visible area where these rays do not intersect the remaining volume). After choosing the best orientation we show it to the user and ask him to orient the part in the specified orientation.

\section{Carving Experiments}

In Figure 6, we show some examples of CAD models and the manual carving process. To perform these experiments, we used a low-resolution Optoma projector (960 X 540 pixels) and a Basler camera (1928 X 1208). The projector and camera were fixed at a certain angle of the workspace table to allow projecting the instructions from several directions.

\section{Future Work}

There are some constraints on the type of CAD models that the method can handle. At first, we expect each part of the model surface to be visible from some direction, otherwise, the algorithm will generate instructions that cannot be performed. One way to handle these cases is to split the CAD model to make its whole surface visible from some angle. Currently, we only compute the best orientation from the six basic orientations. But in the future, we want to develop a more complex algorithm that selects the best orientation in general. 
(1)
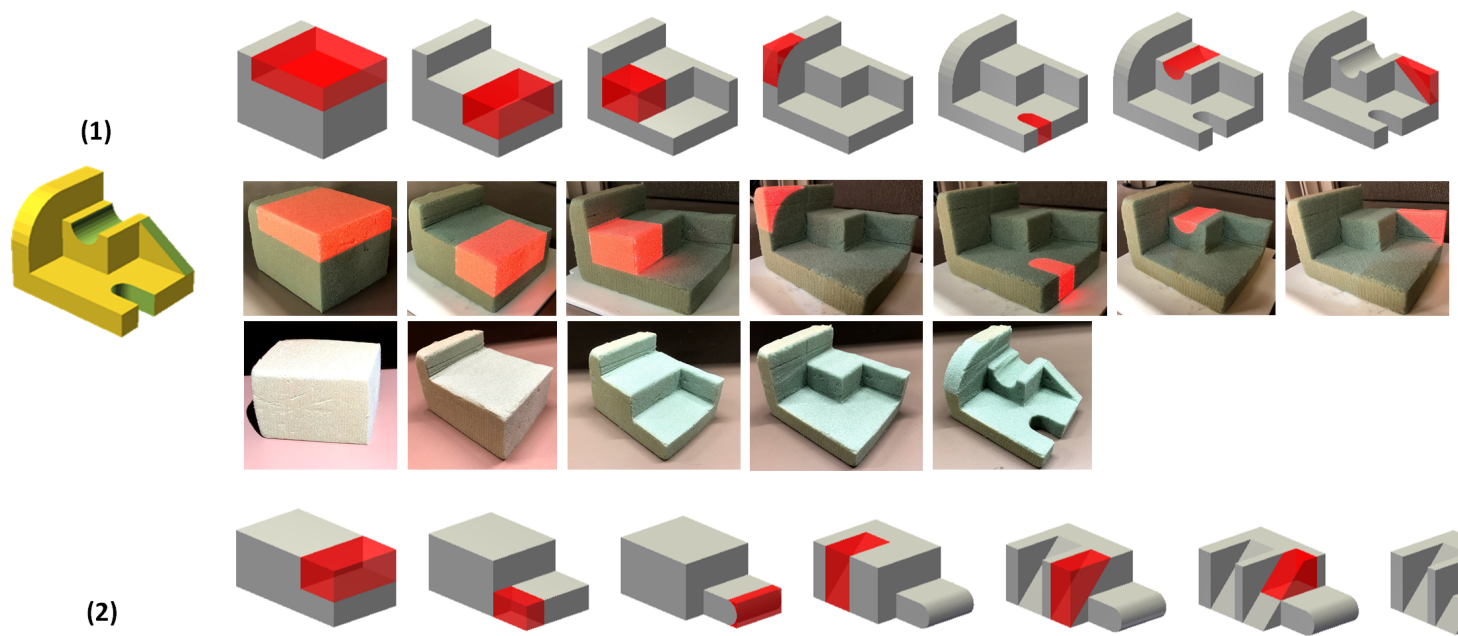

(2)
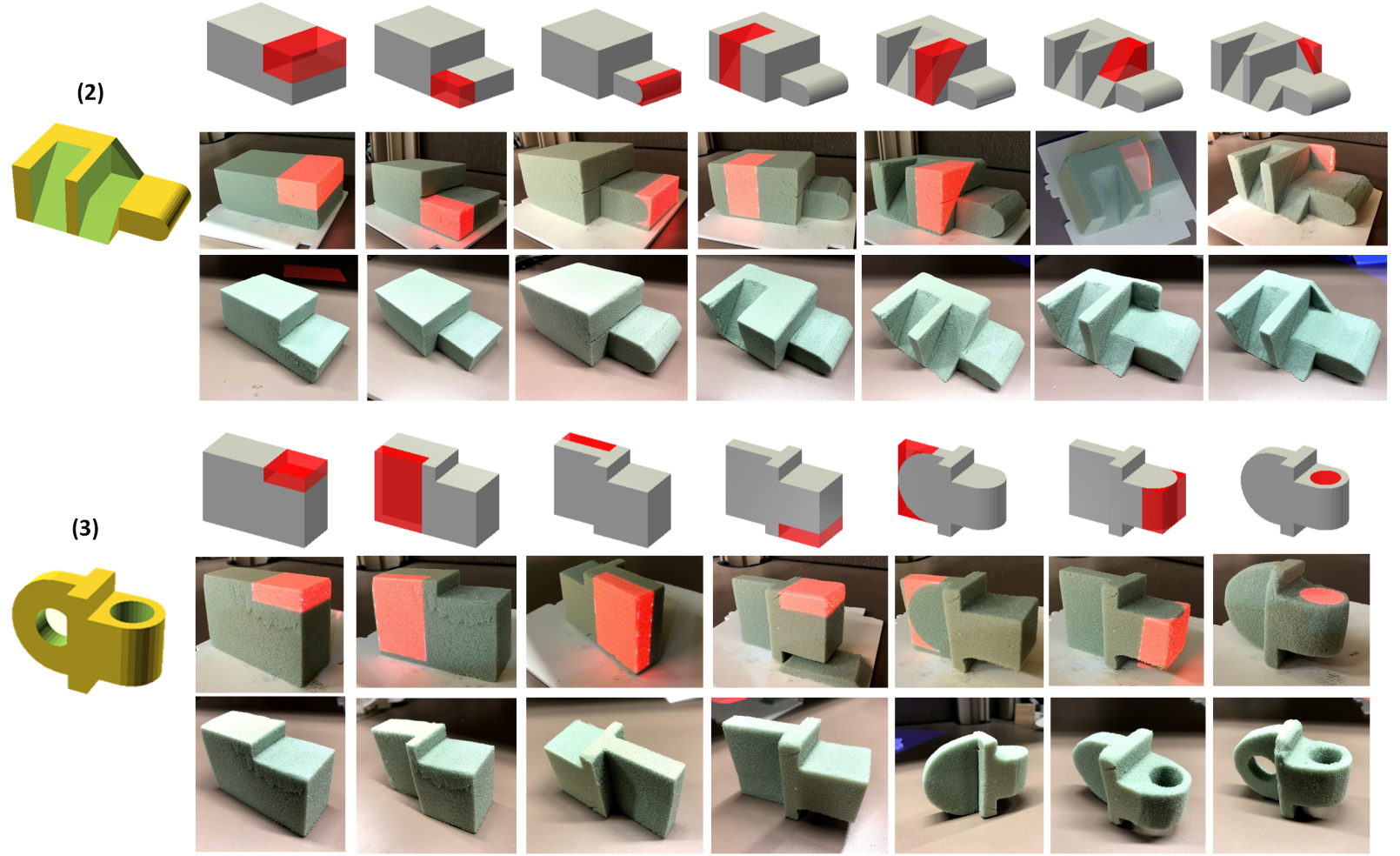

(4)
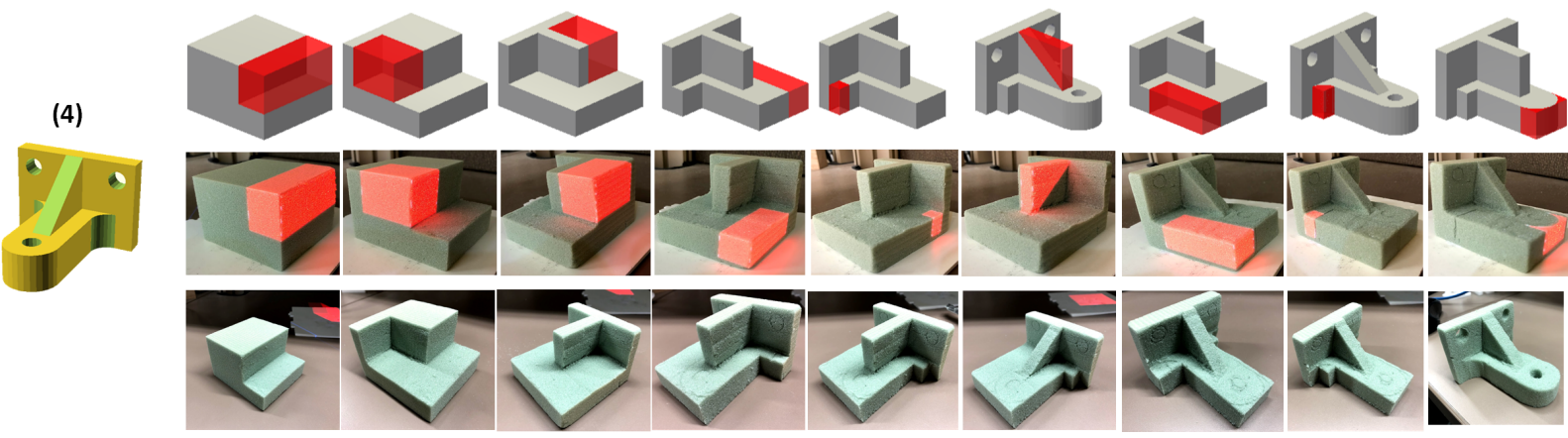

Figure 6. Some examples of CAD models and their manual carving process, with the rendered instructions on the top row, projected instructions on the middle row and the actual cutting result on the bottom row. 
That algorithm should also take into account that we want the model to stand by itself while projecting this orientation.

Besides the visibility constraints, we should order the instructions such that we minimize the number of times where the user must move the object. This will help the user in performing multiple instructions at once. Another limitation of the method is that it does not always match the user preferences. Sometimes there are multiple possible good segmentations, and in general, the user might have his own preference, and he might not like the instructions given. Some other times the instructions given are too long for the user. To help with that, we give the user the option to combine multiple instructions, to skip instructions, or to change the order of instructions. In the future, we want to allow the user to do his own special cuts, and then rerun the algorithm starting from the user operation.

\section{CONCLUSION}

In this preliminary work, we demonstrated a system that helps unskilled users in carving CAD models. We noticed that carving is a hard process for novice users, as they need help figuring out the steps to cut a specific 3D model and to measure and mark the locations to perform these cuts. We presented a method to generate carving instructions for an input CAD model and used a projector to project these instructions to a block of material. To generate the instructions, we need to solve a segmentation problem of the negative space of the CAD model. The method must take into account the visibility constraints and some other factors. 3D alignment is an important step in the system that prepares the system for projecting the next instruction. The system flexibility helps the user to match his preference in merging instructions, changing their order or defining custom instructions.

\section{ACKNOWLEDGMENTS}

The work described herein was partially supported by a Brown Fellowship and by NSF grants IIS-1717355 and IIP-1500249.

\section{REFERENCES}

[1] Paul J Besl and Neil D McKay. 1992. Method for registration of 3-D shapes. In Sensor Fusion IV: Control Paradigms and Data Structures, Vol. 1611. International Society for Optics and Photonics, 586-607.

[2] Oliver Bimber and Ramesh Raskar. 2005. Spatial augmented reality: merging real and virtual worlds. CRC press.

[3] YC Chang, JM Pinilla, JH Kao, J Dong, K Ramaswami, and FB Prinz. 1999. Automated layer decomposition for additive/subtractive solid freeform fabrication. In Proceedings of the Solid Freeform Fabrication Symposium, The University of Texas at Austin. 111-120.

[4] Matthew Flagg and James M Rehg. 2006. Projector-guided painting. In Proceedings of the 19th annual ACM symposium on User interface software and technology. ACM, 235-244.

[5] JungHyun Han, Mike Pratt, and William C Regli. 2000. Manufacturing feature recognition from solid models: a status report. IEEE Transactions on Robotics and Automation 16, 6 (2000), 782-796.
[6] Ammar Hattab, Ian Gonsher, Daniel Moreno, and Gabriel Taubin. 2017. Differential 3D Scanning. IEEE computer graphics and applications 38, 3 (2017), 43-51.

[7] Michael R Marner and Bruce H Thomas. 2010. Augmented foam sculpting for capturing 3D models. In $3 D$ User Interfaces (3DUI), 2010 IEEE Symposium on. IEEE, 63-70.

[8] Daniel Moreno and Gabriel Taubin. 2012. Simple, accurate, and robust projector-camera calibration. In $3 D$ Imaging, Modeling, Processing, Visualization and Transmission (3DIMPVT), 2012 Second International Conference on. IEEE, 464-471.

[9] Stefanie Mueller, Pedro Lopes, and Patrick Baudisch. 2012. Interactive construction: interactive fabrication of functional mechanical devices. In Proceedings of the 25th annual ACM symposium on User interface software and technology. ACM, 599-606.

[10] Huaishu Peng, Jimmy Briggs, Cheng-Yao Wang, Kevin Guo, Joseph Kider, Stefanie Mueller, Patrick Baudisch, and François Guimbretière. 2018. RoMA: Interactive Fabrication with Augmented Reality and a Robotic 3D Printer. (2018).

[11] Alec Rivers, Andrew Adams, and Frédo Durand. 2012a. Sculpting by numbers. ACM Transactions on Graphics (TOG) 31, 6 (2012), 157.

[12] Alec Rivers, Ilan E Moyer, and Frédo Durand. 2012b. Position-correcting tools for 2D digital fabrication. ACM Transactions on Graphics (TOG) 31, 4 (2012), 88.

[13] Ruwen Schnabel, Roland Wahl, and Reinhard Klein. 2007. Efficient RANSAC for point-cloud shape detection. In Computer graphics forum, Vol. 26. Wiley Online Library, 214-226.

[14] Eldon Schoop, Michelle Nguyen, Daniel Lim, Valkyrie Savage, Sean Follmer, and Björn Hartmann. 2016. Drill Sergeant: Supporting physical construction projects through an ecosystem of augmented tools. In Proceedings of the 2016 CHI Conference Extended Abstracts on Human Factors in Computing Systems. ACM, 1607-1614.

[15] Kyle Simek. 2013. Calibrated Cameras in OpenGL. http://ksimek.github.io/2013/06/03/calibrated_ cameras_in_opengl. (2013). Accessed: 2018-04-12.

[16] Christopher Skeels and James M Rehg. 2007. ShapeShift: A projector-guided sculpture system. In Proc. UIST, Vol. 7.

[17] Karl DD Willis, Cheng Xu, Kuan-Ju Wu, Golan Levin, and Mark D Gross. 2011. Interactive fabrication: new interfaces for digital fabrication. In Proceedings of the fifth international conference on Tangible, embedded, and embodied interaction. ACM, 69-72.

[18] Amit Zoran, Roy Shilkrot, and Joseph Paradiso. 2013. Human-computer interaction for hybrid carving. In Proceedings of the 26th annual ACM symposium on User interface software and technology. ACM, 433-440. 